\title{
Condições de trabalho e saúde psíquica: um estudo em dois hospitais universitários ${ }^{1}$
}

\author{
Working conditions and mental health: a study in two
}

university hospitals.

\author{
》 Maria Teresa Pires $\operatorname{COSTA}^{2}$ (Universidade Federal do Rio Grande do Norte, Brasil) \\ » Livia de Oliveira BORGES 3 (Universidade Federal de Minas Gerais, Brasil) \\ » Sabrina Cavalcanti BARROS ${ }^{4}$ (Universidade Federal de Minas Gerais, Brasil)
}

\section{Resumo}

Foi avaliada a relação entre saúde psíquica e condições de trabalho de profissionais de saúde em dois hospitais universitários. Adotou-se uma abordagem psicossociológica, considerando que a compreensão histórico-social do contexto contribui para entender os fenômenos. As condições de trabalho e a saúde psíquica foram abordadas interdisciplinarmente, fundamentando a construção de um modelo compreensivo de saúde psíquica que orientou a pesquisa. Foi desenvolvida aplicação de questionários estruturados e observação participante sobre condições de trabalho e saúde psíquica. A análise dos resultados mostrou que os escores sintomáticos de saúde psíquica variam segundo as condições de trabalho. Os resultados corroboraram que, quanto mais desfavoráveis são as condições de trabalho, mais deteriorada será a saúde psíquica e os afetos com relação ao trabalho, repercutindo novamente no ambiente de trabalho. Portanto, ações de melhoria das condições de trabalho precisam ser estabelecidas para resultar no efeito inverso, proporcionando o aumento dos afetos positivos e a redução dos sintomas psíquicos.

Palavras-chave:

saúde psíquica; condições de trabalho; profissionais de saúde.

\begin{abstract}
This study examined the relationship between psycho health and work conditions to health professionals in two universitary hospitals. The psychosociological approach was adopted, considering that the social and historical comprehension of context contributes to understand the phenomena. The work conditions and the psycho health were approached interdisciplinary and they substantiate the construction of a comprehensive model of mental health that guided the research. The development of this required application of structured questionnaires participant observation about work condition and psycho health. The analysis of results showed that the symptomatic scores vary in according to work conditions. The results corroborated that how more the work conditions are unfavorable, the psycho health and affections with respect to work will be worse. They impact other time in work environment. Then, action to improve work conditions need to be established to generate the inverse effect, facilitating the increase of positive affections and the reduction of psycho symptom. Keywords:

mental health; work conditions; health profession.
\end{abstract}

Resumen

En este estudio se evaluó la relación entre salud psíquica y las condiciones de trabajo de profesionales de salud en hospitales universitarios. Se adoptó una aproximación psicosociológica, considerando que la comprensión histórica-social del contexto contribuye para entender los fenómenos. Las condiciones de trabajo y la salud psíquica fueron abordadas de forma interdisciplinar, fundamentando la construcción de un modelo comprensivo de salud psíquica que orientó la investigación. Para el desarrollo de la misma, se aplicaron cuestionarios estructurados y observación participante sobre las condiciones de trabajo y de salud psíquica. El

1 Pesquisa desenvolvida com o apoio do CNPq na forma de bolsa de produtividade e concessão de recursos por meio de edital universal, bem como com o apoio do Programa de Pós-graduação em Psicologia da UFRN e do Programa de Pós-graduação em Psicologia da UFMG. E-mail: teresapires.psi@gmail.com.

2 Professora Adjunta da Universidade Federal do Rio Grande do Norte, Departamento de Ciências Administrativas.

3 Professora Titular da Universidade Federal de Minas Gerais, Programa de Pós-graduação em Psicologia. Pesquisadora CNPq (PQ1B).

4 Doutoranda do Programa de Pós-graduação em Psicologia da Universidade Federal de Minas Gerais. 
análisis de los resultados demostró que los puntajes de los síntomas de salud psíquica varían de acuerdo a las condiciones de trabajo. Los resultados corroboran que, cuanto más desfavorables son las condiciones de trabajo, más se deteriora la salud psíquica y los afectos con relación al trabajo, lo cual repercute nuevamente en el ambiente de trabajo. Por lo tanto, acciones que mejores estas condiciones necesitan ser establecidas para resultar en el efecto inverso, proporcionando el aumento de los afectos positivos y la reducción de los síntomas psíquicos.

Palabras-clave:

salud psíquica, condiciones de trabajo, profesionales de la salud. 
$\mathrm{E}$ sta pesquisa objetivou avaliar a relação entre saúde psíquica e condições de trabalho de profissionais de saúde em dois hospitais universitários federais do Rio Grande do Norte. Foi fundamentada em uma perspectiva psicossociológica, na qual estudar os fenômenos psicossociais requer considerar a conduta humana em diferentes níveis de análise (pessoal, organizacional e social), tomando-os indissociáveis (Álvaro, Garrido, \& Torregrossa, 2007; Zanelli, Bastos, \& Rodrigues, 2014); assumir o caráter histórico, multidimensional e multideterminante (Álvaro, 1995); e pressupor o papel estruturante do trabalho para a vida humana e para a sociedade (Engels, 1845/2008; Marx \& Engels, 1846/1981).

Adotou-se também como pressuposto a noção de existência como precedente à essência (Politzer, Besse, \& Caveing, 1970; Sartre \& Ferreira, 1961). A consciência é construída na existência, significando que o plano material precede o psíquico e o social. Essas considerações conduzem à explicação do fenômeno sob múltiplas formas de análise e à compreensão em sua totalidade (Álvaro, 1995). Assim, conhecer as condições sob as quais o trabalho é realizado pelos profissionais de saúde torna-se fundamental para compreender os impactos do contexto hospitalar na saúde psíquica desses trabalhadores.

\section{Condições de trabalho}

Uma das características do trabalho hospitalar é lidar com as expectativas do usuário: o desejo de jamais precisar de um hospital (ninguém almeja adoecer), o fato de as instituições de saúde serem socialmente percebidas como um lugar de dor e morte (Pitta, 1990; Silva, 2002), e de os hospitais serem locais de exclusão social, visto que o hospitalizado fica temporariamente fora do sistema produtivo, contradizendo a função dessa instituição de ser um local de recuperação e reinserção social.

Quando um usuário procura um hospital, há expectativa de se sentir excluído do meio social e do papel de trabalhador, ainda que por um curto período de tempo (Castelhano, 2005). Isso interfere nas relações estabelecidas por ele com os profissionais de saúde, passando a representar aspectos das condições de trabalho destes. Ademais, existem as expectativas desses profissionais, que também afetam suas condições de trabalho e psiquismo, porque contribuem para construir e caracterizar o ambiente psicossocial do hospital.

O trabalho hospitalar (Pires, 2000) sobrevaloriza as especificidades das atividades e as qualificações profissionais exigidas; naturaliza a falta de parcerias entre os profissionais, aumentando esforços e contribuindo para medidas contraditórias na assistência prestada; e é atravessado por relações de poder, cuja dominação do saber médico interfere nos processos das atividades em saúde e no modo de atuação de outros profissionais de saúde (enfermeiros, fisioterapeutas, nutricionistas, entre outros). Merhy (1999) e Schraiber et al. (1999) ressaltaram que a organização dos serviços de saúde obedece uma lógica neoliberal que afeta a missão e o uso da tecnologia, resultando na adoção de políticas que desprotegem o trabalhador, interferindo na realização de suas atividades.

Segundo Schraiber etal. (1999), a divisão do trabalho na área da saúde deveria resultar na complementaridadee interdependência dos trabalhos especializados. Entretanto, em uma equipe multiprofissional, quando os agentes possuem autoridades desiguais, a complementaridade e a interdependência se opõem. Consequentemente, esses agentes buscam a ampliação apenas da autonomia técnica quando deveriam articulá-las a ações de cunho político e estratégico para obterem eficácia e eficiência.

Ramos, Peiró e Ripoll (2002), Blanch (2003), Garrido (2005), Peiró e Prieto (2002) e Prieto (1994) têm destacado a necessidade de sistematizar os conteúdos que compõem as condições de trabalho, bem como a de incorporar o enfoque político. Atribuem a esse fenômeno um conjunto de aspectos que abrange o entorno físico, o conteúdo das atividades, as características do ambiente de trabalho e as condições sociais em que se desenvolve. Tal compreensão sobre o fenômeno tem sido adotada por outros pesquisadores (Joyce, Pabayo, Critchley, \& Bambra, 2010; McCue, 1982; Sanz Yagüez \& López Corbalán, 1999), relacionando-o com aspectos do trabalho como satisfação, segurança e prevenção de riscos laborais.

Ademais, o European Working Conditions Observatory [EWCO] (2006) tem monitorado as condições de trabalho dos europeus, utilizando um instrumento cujas questões se aproximam das taxonomias propostas por Ramos et al. (2002), Blanch (2003), Peiró e Prieto (2002) e Garrido (2005). Borges, Alves-Filho, Costa e Falcão (no prelo) as sintetizaram, abrangendo quatro categorias (Tabela 1). 
TABELA 1. As grandes categorias das condições de trabalho (taxonomia).

\section{Categorias}

1) Condições contratuais e jurídicas

\section{2) Condições físicas e} materiais

\section{3) Processos e características} da atividade

\section{4) Condições do ambiente sociogerencial}

\section{Conceitos}

Conjunto de aspectos jurídicos (autônomo versus emprego), contratuais, no caso do emprego (formal ou informal), de estabilidade do contrato (instável ou estável) e de modalidades de contrato formal (temporário, por tempo indefinido). Abrange também aspectos como o sistema de incentivo (retribuições) e as definições do tempo a ser dedicado ao trabalho.

São os componentes mais concretos. Referem-se ao entorno das atividades de trabalho no que diz respeito às condições físicas, ao espaço arquitetônico e a instalações, às condições de segurança física e/ou material e às formas com que se lida com o impacto do espaço geográfico e as condições climáticas sobre o trabalho.

Abrange aspectos relativos ao conteúdo das atividades de trabalho, à organização e divisão do trabalho, às demandas do posto de trabalho, aos modos de execução das atividades e ao desempenho do trabalhador.

Aspectos relacionados às interações interpessoais (sejam horizontais ou verticais), às práticas sociais relativas à gerência ou à gestão, quando se trata do trabalho na forma de emprego (pois que as condições de trabalho adquirem uma dimensão organizacional), e às práticas sociais decorrentes da inserção no mercado de trabalho (parcerias, redes de trabalho formais ou informais, etc.).

Fonte: Borges et al. (no prelo)

As subcategorias podem ter sentidos e organizações distintas por ocupação, como Borges et al. (2013) mostraram com operários da construção civil, docentes e profissionais de saúde. A Tabela 2 apresenta, então, os fatores encontrados para docentes e profissionais de saúde para três das dimensões referidas.

TABELA 2. Fatores das dimensões de condições de trabalho de docentes e profissionais de saúde.

Fatores
Aspectos psicobiológicos
Espaço de trabalho
Aspectos fisicoquímicos
Exigências de esforço fisico
Riscos de acidentes
Espaço de autonomia
Complexidade, responsabilidade
e rapidez

Organização do tempo

Estímulo à colaboração

\section{Condições físicas e materiais}

Perceber-se exposto a riscos do ambiente físico e material que podem ter impacto na saúde (corporal e psíquica).

Perceber-se exposto a realizar as atividades dentro de instalações específicas da organização e/ou quanto é necessário ser realizado no campo e no espaço virtual.

Perceber-se exposto a aspectos do ambiente físico e material do trabalho, como presença de vapores, fumaça e poeira, temperatura e iluminação.

Perceber-se exposto a atividades de trabalho de execução mecânica e movimentos repetitivos, com uso de máquinas e equipamentos.

Percebe-se exposto a diferentes riscos de acidentes de pequeno porte, incapacitantes e fatais.

\section{Processos e características da atividade}

Na organização do trabalho, contar com a possibilidade de decidir de forma autônoma pelos métodos, planejamento e ritmo do que se realiza. Espaço de ação.

Quanto se exige do trabalhador execução de tarefas complexas, rapidez, responsabilidade por danos e iniciativa diante do imprevisto.

Quanto de autonomia se dispõe para organizar o próprio trabalho no tempo, planejar intervalos, folgas e férias.

Quanto é possível contar com a colaboração dos pares e colaborar com eles na realização do trabalho.

\section{Condições do ambiente sociogerencial}

Organização das atividades

Refere-se à natureza e à distribuição das atividades no ambiente de trabalho. Revela o aspecto da percepção do participante sobre o exercício da função gerencial organizativa, o que justifica a denominação.

Refere-se a pressões diretas e indiretas (por meio da fragilidade infraestrutural):

Infraestrutura e pressão exposição a situações de falta de equipamentos e material de trabalho e percepção de exigências desproporcionais às condições de infraestrutura.

Diz respeito à percepção dos participantes sobre ações gerenciais de prevenção a

Oferta de informação de saúde acidentes de trabalho e a problemas de saúde ocupacionais, informando o trabalhador sobre os riscos existentes.

Refere-se à sujeição dos participantes a situações de discriminação baseadas em traços pessoais (idade, altura, cegueira, sexo, etc.), fazendo parte da dimensão sobre o ambiente sociogerencial alude. Portanto, a percepção do participante quanto ao gerenciamento na organização que minimiza ações discriminatórias. 


\begin{tabular}{|c|c|}
\hline Participação & $\begin{array}{l}\text { Itens referentes à percepção dos participantes sobre as práticas interativas de } \\
\text { consulta sobre mudanças na organização do trabalho e de abertura ao diálogo em } \\
\text { torno do desempenho no trabalho. }\end{array}$ \\
\hline Violência & $\begin{array}{l}\text { Itens que aludem a ameaças de violência físicas, agressões verbais, intimidações, } \\
\text { perseguições e discriminação sexual. Refere-se, portanto, à amistosidade das } \\
\text { relações interpessoais. }\end{array}$ \\
\hline Ambiente conflitante & $\begin{array}{l}\text { Refere-se à percepção do participante sobre as possibilidades de ser envolvido em } \\
\text { conflitos interpessoais e em situações que divergem de seus valores e princípios. }\end{array}$ \\
\hline
\end{tabular}

Fonte: Reeditado de Borges et al., 2013.

Em concordância com os estudos citados (Blanch, 2003; Garrido, 2005; Peiró \& Prieto, 2002; Prieto, 1994; Ramos et al., 2002), esta pesquisa parte da concepção adotada por Blanch, para quem condições de trabalho representa um conjunto de circunstâncias sob as quais se desenvolvem as atividades laborais e incidem significativamente na experiência laborativa e nas dinâmicas das relações estabelecidas no ambiente de trabalho. Optou-se por utilizar as taxonomias sintetizadas em quatro grandes categorias (Tabela 1), a partir das quais Borges et al. (2013) desenvolveram pesquisas considerando as especificidades ocupacionais do país.

\section{Saúde psíquica e trabalho em saúde}

A relação entre doença, trabalho, normalidade e patologia é uma preocupação retratada no desenvolvimento das ciências e atravessa os discursos e/ou concepções contemporâneas sobre saúde (Batistella, 2007; Coelho \& Almeida Filho, 2002). Os modelos que se propuseram a compreender esse fenômeno apresentaram diferentes abordagens explicativas sobre a saúde e seu impacto psicológico nos indivíduos. Tais modelos partiram de uma concepção de saúde representando ausência de doença, bem-estar, dimensão normativa da vida, e direito do cidadão.

A saúde concebida como ausência de doença pauta-se na cura. Buscam-se sinais de doença e, na ausência destes, toma-se o indivíduo como saudável. A ênfase em evidências empíricas de saúde a partir da não doença estabelece um conceito negativo dela (Leriche, 1940), sendo criticado por Parsons (1951), que considerou a doença como algo inerente à condição humana e, portanto, parte constituinte da norma social.

A formulação da Organização Mundial da Saúde [OMS] (1948) sobre a saúde enfatiza o completo bemestar físico, mental e social do indivíduo. Representa um avanço ao tratar a saúde de maneira mais positiva ao considerar o bem-estar do sujeito, embora a crítica a este modelo o considere utópico e pautado em uma noção subjetivista de bem-estar (Almeida \& Jucá, 2002; Benavides, García \& Ruiz-Frutos, 2000). Recentemente, a OMS (2001) adotou um conceito de saúde psíquica que engloba o bem-estar subjetivo, definindo critérios para a saúde mental (por exemplo, crescimento, desenvolvimento e autorrealização, integração e resposta emocional, domínio ambiental e competência social).

Outros autores (Gouveia, Fonseca, Lins, \& Gouveia, 2008; Gouveia, Lins, Lima, Freires, \& Gomes, 2009; Warr, 1987) propuseram diferentes abordagens para o bem-estar afetivo no trabalho, relacionando-o à saúde psíquica e considerando-o decorrente das percepções do indivíduo sobre seu contexto laboral. Para Gouveia et al. (2009), o bem-estar subjetivo refere-se ao modo que os indivíduos avaliam suas vidas, variando individualmente, pois depende de expectativas, valores e experiências anteriores. Os afetos, segundo esses autores, traduzem valências positivas ou negativas sobre o trabalho, levando a níveis de ativação opostos (excitação e letargia).

A concepção de saúde como uma construção social a caracteriza como uma dimensão normativa da vida, cujas regras são estabelecidas frente às diversas situações do cotidiano. O homem saudável é normativo em relação às demandas do meio, sendo, portanto, normal do ponto de vista do processo saúde-doença. A incapacidade de se ajustar às demandas do meio, por sua vez, conduz à patologia, resultando em sintomas como sofrimento, contrariedade, impotência diante da vida e alterações funcionais (Canguilhem, 1966/2006).

Canguilhem não considera anormal e patológico como sendo sinônimos. Se o fenômeno observado for fora da norma geral, mas apresentar funcionalidade para o sujeito, então não podemos dizer que não há saúde. Contudo, se há disfunção resultando em sofrimento e sintomas, estamos diante de uma condição patológica.

A crise dos sistemas de saúde nas décadas de 1970 e 1980 provocou mobilização nos países latinos, ampliando, no Brasil, o conceito de saúde e caracterizando-a na Constituição Federal (Art. 196) como um direito de todos e um dever do Estado, cabendo a ele ações para reduzir o risco de doenças e ampliar o acesso 
igualitário às ações preventivas (Constituição, 1988). Tal concepção evidencia a necessidade de mobilização das esferas sociais para que o direito à saúde seja garantido a todos.

No entanto, historicamente, o cuidado com a saúde tem sido primeiramente uma obrigação familiar, seguida pelo trabalho preventivo de medidas higiênicas escolares e pelo cuidado da saúde do trabalhador nas organizações, como prescrições legais. Neste último, as práticas têm focalizado os acidentes e as doenças ocupacionais, deixando de considerar a integralidade do ser humano trabalhador (Mozer \& Kherig, 2006).

Segundo o Ministério da Saúde [MS] (2001), há uma evolução legal da assistência à saúde do trabalhador no Protocolo de Procedimentos Médico-Periciais no 5.1, que reconhece que o nexo causal de um agravo ocorre entre a doença e o trabalho, buscando-se, portanto, um ente nosológico. O Conselho Federal de Medicina [CFM] (1998), no Art. 2º da Resolução CFM 1488/98, recomenda ao médico perito buscar, além do exame clínico físico e mental, exames complementares. Abriu-se, então, espaço para a constituição do Nexo Técnico Epidemiológico (NTEP) e para as intervenções sustentadas na compreensão da relação do trabalhador com o seu contexto de trabalho.

A abordagem psicossociológica, como já exposto, pauta-se na interdisciplinaridade e na possibilidade de articulação dos vários níveis de análise, permitindo a compreensão do nexo entre saúde e trabalho. A partir disso, formulou-se um modelo compreensivo de saúde contemplando condições de trabalho; riscos inerentes ao trabalho; aspectos biológicos, psicológicos e sociológicos; e ações de garantia do direito à saúde (Figura 1).

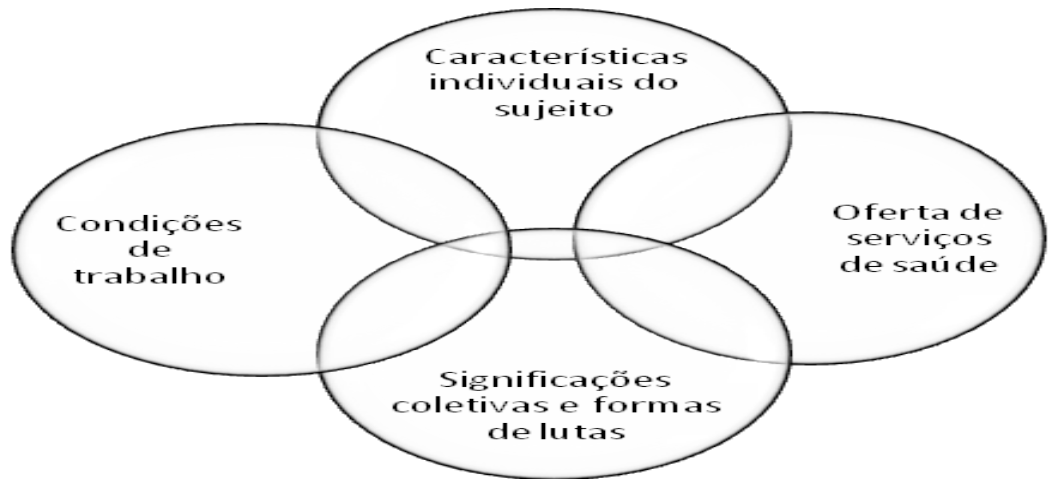

FIGURA 1. Modelo compreensivo de saúde psíquica no trabalho

A saúde no trabalho resulta da interseção entre as características individuais dos sujeitos possuidores de uma história e de características biopsicossociológicas; as condições de trabalho compreendidas como relações no trabalho, a organização do processo de trabalho e as condições em que o sujeito exerce suas atividades; a oferta de ações nos níveis de prevenção, atenção e reabilitação; e as crenças do coletivo, expressas nas estratégias de luta adotadas em prol da própria saúde.

\section{MÉTODO}

A pesquisa foi desenvolvida em dois hospitais da Universidade Federal do Rio Grande do Norte (UFRN), sendo um geral (HG), e outro, materno-infantil (HMI). As estratégias utilizadas foram aplicação de questionários estruturados e observação participante.

Os participantes eram profissionais de saúde - enfermeiros, médicos, odontólogos e assistentes sociais que prestam assistência ao usuário dos serviços e mantêm vínculo empregatício com a UFRN. Formou-se uma amostra acidental de 120 participantes, cuja acessibilidade e voluntariedade foram os critérios básicos de inclusão.

Dos instrumentos, o Questionário de Condições de Trabalho (QCT) teve suas evidências de validade e consistência examinadas com uma amostra de profissionais de saúde e docentes de ensino superior da qual os participantes desta pesquisa tomaram parte (Borges et al., 2013). Seus itens estão organizados segundo as categorias identificadas na Tabela 1. Em referência, a categoria Condições Contratuais e Jurídicas as questões são estruturadas, mas suas respostas não escalares. Entretanto, as suas respostas não serão utilizadas nas análises deste artigo porque todos os participantes vinculam-se a um mesmo empregador e, consequentemente, tais respostas apresentaram-se aproximadamente constantes. Sobre as demais categorias, o QCT mensura os fatores definidos na Tabela 2 . 
O Questionário de Saúde Geral - 60 (QSG-60) foi adaptado para o Brasil por Pasquali, Gouveia, Andriola, Miranda e Ramos (1994) e mensura seis fatores: estresse psíquico, desejo de morte, desconfiança do próprio desempenho e autoeficácia, distúrbio do sono, distúrbio psicossomático e saúde geral, referente à soma dos fatores anteriores, representando a presença ou a ausência dos distúrbios psiquiátricos não psicóticos. Os coeficientes alfa de Cronbach variam de 0,80 a 0,89 e, no fator geral, atinge 0,95.

A Escala de Bem-Estar Afetivo no Trabalho (JAWS) foi adaptada por Gouveia et al. (2008) e avalia o bemestar subjetivo no trabalho. Utiliza como critérios a valência positiva e o nível de excitação, mensurando quatro fatores que derivam da combinação dos critérios citados. Aplicou-se, ainda, uma ficha sociodemográfica.

Desenvolveu-se a aplicação dos questionários na seguinte sequência: contato com a direção dos hospitais da UFRN, solicitando a autorização para abordar os profissionais; contato com carta de apresentação da pesquisa firmada pelas pesquisadoras e pelo dirigente da organização/instituição; contato com os participantes, solicitando a sua adesão voluntária à pesquisa; aplicação do Termo de Consentimento Livre e Esclarecido (TCLE); e aplicação do conjunto de questionários. A aplicação foi individual, obedecendo às especificidades do campo de pesquisa e do grupo pesquisado. Preservou-se o anonimato dos participantes e, em virtude disso, o TCLE foi arquivado separadamente, desvinculando o termo do protocolo de pesquisa respondido.

Para análise das condições de trabalho, estimou-se os escores nos fatores do QCT, calculou-se a média e o desvio padrão, e, por fim aplicou-se a ANOVA para medidas repetidas e com teste post hoc Bonferroni. Para análise da saúde psíquica dos participantes, foram calculados os percentis sintomáticos das respostas ao questionário QSG-60, distribuídos em três níveis (sem sintomas, sintomático e sintomas graves) e estimados os escores das dimensões da JAWS-12por meio da média dos pontos que os respondentes atribuíram aos itens que compõem cada dimensão. Os escores nos fatores do QSG-60 e JAWS foram agrupados (análise de clusters), identificando conglomerados da saúde psíquica, e foi avaliada a variância dos escores nos fatores do QCT por esses agrupamentos utilizando a ANOVA.

Este artigo é um recorte de uma pesquisa mais ampla. Diante da necessidade de concisão, os resultados da observação participante serão apresentados parcialmente, apenas referindo-se ao indispensável para apoiar as análises das respostas aos questionários estruturados. Na pesquisa mais ampla, a interdependência das duas técnicas (questionários estruturados e observação participante) está posta e torna-se mais evidente nos resultados. Por consequência de tal interdependência, foi impossível omiti-la por completo neste artigo.

A observação participante foi realizada pela primeira autora, imersa no contexto de pesquisa durante 90 dias. Consistiu na interação entre ela e os participantes, tornando tênues os limites entre formalidade e informalidade. A informalidade resultou da espontaneidade que fez parte dos atos interativos de pesquisador e participantes. O objetivo foi apreender informações que emergiram, em tal contexto, da espontaneidade. Os dados desta observação foram registrados em diários de campo.

\section{RESULTADOS E DISCUSSÃO}

\section{Condições de trabalho}

Em referência às condições físicas e materiais (Tabela 3), as diferenças entre as médias são acentuadas e os participantes percebem que há maior exposição aos aspectos psicobiológicos do trabalho, seguidos pela exigência de esforço físico.

A literatura sobre condições de trabalho em saúde (por exemplo: Costa, Lima, \& Almeida, 2003; Elias \& Navarro, 2006; Murofuse, Abranches, \& Napoleão, 2006) aponta que os hospitais são locais de risco à saúde do trabalhador em decorrência das condições de trabalho. A exposição aos riscos psicobiológicos existe devido às ações assistenciais e seu nível de complexidade. A média dos escores no fator $(\mathrm{M}=3,36)$ reflete isso, indicando que os participantes consideram que sua exposição a esses fatores acontece entre algumas vezes e muitas vezes.

Sobre o fator "Exigência de esforço físico", assinala-se que o trabalho em saúde exige do trabalhador movimentos e posturas que levam a situações de desgaste. Este aspecto foi considerado para o estabelecimento legal da redução da jornada de trabalho de algumas categorias profissionais, como fisioterapeutas e odontólogos. Autores como Laurell e Noriega (1989) reconheceram esta condição, que é normatizada pelo Ministério do Trabalho e Emprego [MTE] (2005) por meio da Norma Regulamentadora de No 32 (NR-32), estabelecendo ações para minimizar os riscos ergonômicos no trabalho em saúde, como as posições dolorosas e fatigantes. 
TABELA 3. Médias nos fatores das dimensões das condições de trabalho $(n=120)$

\begin{tabular}{|c|c|c|c|c|c|c|}
\hline \multirow{2}{*}{ Fatores } & \multirow{2}{*}{ Média } & \multirow{2}{*}{$\begin{array}{l}\text { Desvio } \\
\text { padrão }\end{array}$} & \multicolumn{4}{|c|}{ Intervalos \% } \\
\hline & & & $x<2$ & $2<x<3$ & $3<x<4$ & $x>4$ \\
\hline \multicolumn{7}{|c|}{ Condições físicas e materiais } \\
\hline F1- Aspectos psicobiológicos & 3,36 & 0,84 & 3,3 & 34,2 & 37,5 & 25,0 \\
\hline F2 - Espaço de trabalho & 2,24 & 0,74 & 41,7 & 42,5 & 14,2 & 1,7 \\
\hline F3 - Aspectos físico-químicos & 1,92 & 0,61 & 57,5 & 37,5 & 5,0 & - \\
\hline F4 - Exigência de esforço físico & 2,81 & 0,84 & 20,8 & 35,8 & 33,3 & 10,0 \\
\hline F5 - Risco de acidentes & 1,88 & 0,70 & 66,7 & 27,5 & 3,3 & 2,5 \\
\hline \multicolumn{7}{|c|}{$F=2754,43$ para $p<0,001$} \\
\hline \multicolumn{7}{|c|}{ Processos e características do trabalho } \\
\hline F1- Espaço de autonomia & 2,81 & 0,05 & 9,2 & 58,3 & 30,8 & 1,7 \\
\hline F2 - Complexidade e responsabilidade & 3,27 & 0,62 & 1,7 & 35,8 & 50,0 & 12,5 \\
\hline F3 - Organização do tempo & 2,55 & 0,06 & 18,3 & 58,3 & 23,3 & - \\
\hline F4 - Estímulo à colaboração & 3,49 & 0,71 & 2,5 & 31,7 & 37,5 & 28,3 \\
\hline ANOVA & \multicolumn{3}{|c|}{$\mathrm{F}=6608,46$ para $\mathrm{p}<0,001$} & & & \\
\hline \multicolumn{7}{|c|}{ Ambiente sociogerencial } \\
\hline F1- Organização das atividades & 2,28 & 0,80 & 44,2 & 34,2 & 17,5 & 4,2 \\
\hline F2 - Infraestrutura e pressão & 3,02 & 0,94 & 18,3 & 38,3 & 30,0 & 13,3 \\
\hline F3 - Oferta de informações de saúde & 2,43 & 0,11 & 50,8 & 16,7 & 21,7 & 10,8 \\
\hline F4 - Discriminação social & 1,16 & 0,35 & 95,8 & 3,3 & 0,8 & - \\
\hline F5 - Participação & 2,46 & 0,70 & 33,3 & 43,3 & 21,7 & 1,7 \\
\hline F6 - Violência & 1,52 & 0,63 & 80,8 & 15,8 & 1,7 & 1,7 \\
\hline F7 - Ambiente conflitante & 2,22 & 0,93 & 55,8 & 30,8 & 6,7 & 6,7 \\
\hline ANOVA & \multicolumn{6}{|c|}{$F=2236,16$ para $p<0,001$} \\
\hline
\end{tabular}

Sobre os processos e as características da atividade, o teste Bonferroni mostrou que a média dos escores dos participantes no fator "Estímulo à colaboração" é mais elevada, o que denota maior exposição ao fator e significa que, neste aspecto, as condições de trabalho são percebidas como adequadas. Entre todos os fatores em análise, este foi o que apresentou maior índice de respostas correspondentes a "frequentemente expostos à condição" (28,3\%). A colaboração entre membros de uma equipe é uma condição desejada para o trabalho em saúde por motivos técnico-assistenciais e pelo apoio psicossocial que pode proporcionar ao trabalhador.

O segundo fator com maior exposição para os participantes foi "Complexidade e responsabilidade". As médias mostram que eles se sentem raramente expostos (30,85\%) e algumas vezes expostos (50\%) a esta condição. Os escores indicam que os participantes consideram as tarefas desempenhadas relativamente complexas e exigem deles responsabilidade. O trabalho em saúde (vide Merhy, 1999; Pires, 2000; Schraiber et al., 1999) é complexo por sua natureza em lidar com vidas, requerendo decisões rápidas e acertadas.

Os fatores "Espaço de autonomia" e "Organização do tempo" tiveram médias no mesmo nível hierárquico e seus resultados indicaram que os profissionais de saúde percebem pouca autonomia no desenvolvimento de seu trabalho. Estudiosos do trabalho e sua relação com a saúde psíquica (Albrecht, 1990; Benevides-Pereira, 2002; Mauro, Mauro, Pinheiro, \& Silva, 2010) estabeleceram a relação entre autonomia e bem-estar psíquico. Entre os participantes da pesquisa, a redução do espaço de autonomia encontrada pode ser um aspecto que predispõe o adoecimento.

Os resultados indicaram também que, para a maioria dos participantes, não é possível ter pleno controle do tempo no trabalho, condições esperadas frente às características da ocupação. A observação participante permitiu perceber que a autonomia e a organização do tempo, embora ocasionais, são mais frequentes entre os profissionais de nível superior do que entre os de nível médio. No nível superior, o espaço de autonomia é maior nas atividades ambulatoriais, em que é comum os profissionais modificarem o agendamento de suas consultas.

Em referência ao ambiente sociogerencial, há acentuada diferença entre as médias dos fatores e maior exposição ao fator "Infraestrutura e pressão". Quanto maior o escore apresentado, mais inadequadas as condições sociogerenciais percebidas pelos participantes, posição de 43,3\% da amostra.

O teste Bonferroni mostrou que os participantes perceberam com a mesma intensidade as condições de trabalho representadas pelos fatores "Oferta de informações de saúde", "Participação" e "Ambiente conflitante". Em relação ao primeiro, observa-se (Tabela 3) que 50,8\% dos participantes informou que nunca ou raramente recebem informações de saúde. Esse resultado é contrastante ao fato de os profissionais de saúde estarem 
expostos a vários riscos ocupacionais que podem levar a acidentes e ao adoecimento. Em razão disso, a NR 32 (MTE, 2005) estabelece medidas de proteção à segurança e à saúde dos trabalhadores dos serviços de saúde, referindo-se aos riscos existentes e à sua prevenção.

No fator "Participação", 76,6\% dos respondentes indicou que as possibilidades de participação são reduzidas. No fator "Ambiente conflitante", tanto a média como a frequência no escore das respostas nos dois primeiros intervalos $(86,6 \%)$ refletem que, para eles, a possibilidade de envolvimento em conflitos interpessoais e divergência de valores é muito baixa, sendo esta uma adequada condição de trabalho. Essas respostas parecem surpreender e ir de encontro ao que foi observado no contexto de pesquisa com a deflagração da greve dos técnico-educativos em educação - categoria ocupacional da qual os profissionais de saúde sujeitos da pesquisa fazem parte -, pois, nesse período, as reclamações e conflitos foram frequentes. No entanto, as respostas são coerentes, mostrando que as reivindicações divulgadas pelo sindicato e na mídia não afetaram a relação com colegas nos diversos níveis hierárquicos. Não há conflito de interesses percebido a partir da análise estatística, nem na observação participante.

A "Organização das atividades" é percebida como adequada pela maioria dos participantes, uma vez que $78,4 \%$ das respostas foram entre nunca e algumas vezes. No fator "Violência", a média obtida $(M=1,52)$ demonstra que os participantes consideram adequadas as condições de trabalho sob esse aspecto, o que é corroborado pela frequência de $96,6 \%$ nos dois primeiros intervalos, cujas respostas significam que o participante nunca está exposto ou está raramente exposto à violência. Já no fator "Discriminação social", as respostas mostram que os participantes da pesquisa $(95,8 \%)$ não se sentem discriminados.

\section{Saúde psíquica e bem-estar afetivo}

No que se refere ao QSG-60 (Tabela 4), em estresse e em desejo de morte, os respondentes apresentam-se mais distribuídos entre os três níveis, havendo maior concentração nos dois últimos, o que sinaliza a presença de ambos na vida desses profissionais. Já nos demais fatores, a distribuição tende a se concentrar nos dois últimos níveis de resposta.

De maneira geral, os participantes apresentam algum nível de dificuldade psicológica, podendo traduzirse como comprometimento do bem-estar psíquico e como dificuldade em manter uma vida pessoal e social satisfatórias.

TABELA 4. Frequência de participantes por fator do QSG e escores nas dimensões da JAWS

\begin{tabular}{|c|c|c|c|c|c|c|}
\hline \multicolumn{7}{|c|}{ QSG-60 } \\
\hline \multirow{2}{*}{ Fatores } & \multirow{2}{*}{$\begin{array}{c}\text { Sem sintomas } \\
\text { Frequência (\%) }\end{array}$} & \multirow{2}{*}{$\begin{array}{c}\text { Sintomáticos } \\
\text { Frequência (\%) }\end{array}$} & \multirow{2}{*}{\multicolumn{2}{|c|}{$\begin{array}{l}\text { Sintomas graves } \\
\text { Frequência }(\%)\end{array}$}} & \multirow{2}{*}{\multicolumn{2}{|c|}{ Total (\%) }} \\
\hline & & & & & & \\
\hline Estresse & $40(33,3)$ & $49(40,8)$ & \multicolumn{2}{|r|}{$31(25,8)$} & \multicolumn{2}{|c|}{$120(100,0)$} \\
\hline Desejo de morte & $40(33,3)$ & $49(40,8)$ & \multicolumn{2}{|r|}{$31(25,8)$} & \multicolumn{2}{|c|}{$120(100,0)$} \\
\hline $\begin{array}{l}\text { Desconfiança no desempenho e } \\
\text { autoeficácia }\end{array}$ & $42(35,0)$ & $61(50,8)$ & \multicolumn{2}{|r|}{$17(14,2)$} & \multicolumn{2}{|c|}{$120(100,0)$} \\
\hline Distúrbio do sono & $21(17,5)$ & $63(52,5)$ & \multicolumn{2}{|r|}{$36(30,0)$} & \multicolumn{2}{|c|}{$120(100,0)$} \\
\hline Distúrbios psicossomáticos & $21(17,5)$ & $63(52,5)$ & \multicolumn{2}{|r|}{$36(30,0)$} & \multicolumn{2}{|c|}{$120(100,0)$} \\
\hline Saúde geral & $37(30,8)$ & $60(50,0)$ & \multicolumn{2}{|r|}{$23(19,2)$} & \multicolumn{2}{|c|}{$120(100,0)$} \\
\hline \multicolumn{7}{|c|}{ JWAS } \\
\hline \multirow{2}{*}{ Fatores } & \multirow{2}{*}{\multicolumn{2}{|c|}{ Média (desvio padrão) }} & \multicolumn{4}{|c|}{ Intervalos (\%) } \\
\hline & & & $x<2$ & $2<x<3$ & $3<x<4$ & $x>4$ \\
\hline \multicolumn{2}{|c|}{ Valência positiva e excitação alta (VPEA) } & $3,45(0,88)$ & 6,7 & 32,5 & 45,0 & 15,8 \\
\hline \multicolumn{2}{|c|}{ Valência positiva e excitação baixa (VPEB) } & $3,61(0,86)$ & 7,5 & 23,3 & 45,0 & 24,2 \\
\hline Valência negativa e excitação bai & VNEA) & $(0,77)$ & 49,2 & 37,5 & 12,5 & 0,8 \\
\hline Valência negativa e excitação bai & VNEB) & $(0,86)$ & 36,7 & 47,5 & 12,5 & 3,3 \\
\hline
\end{tabular}

No JAWS-12 (Tabela 4), a maior média se encontra na dimensão Valência Positiva e Excitação Baixa (VPEB), havendo uma tendência dos profissionais de saúde de apresentar mais sentimentos de tranquilidade, contentamento e satisfação para com o trabalho. A menor média, por sua vez, está presente no fator Valência Negativa Excitação Baixa (VNEB), indicando serem menos frequentes sentimentos de raiva, incômodo e fúria com relação ao trabalho. Isso significa que os sentimentos negativos não são capazes de provocar ativação do comportamento do sujeito, mas nem por isso são menos danosos ao bem-estar psíquico do indivíduo, que provavelmente sofre em silêncio. Seus sentimentos irão repercutir em seu ambiente de trabalho, já que este é simultaneamente físico e relacional. 
Canguilhem (1966/2006) defende que saúde é um regulador das reações dos atores sociais ao meio. Assim, indicadores de comprometimento de saúde e bem-estar psíquicos significam que a capacidade de ajustamento dos indivíduos diminuiu, afetando dialeticamente a relação entre esses atores, seja no trabalho, seja na vida privada. Então, os participantes agruparam-se conforme a afetação de sua saúde psíquica, não apresentando uma hierarquia de severidade, mas traduzindo sua percepção com relação a ela. A análise de clusters foi realizada a partir do escore dos fatores no QSG-60 e na JAWS, sendo identificados cinco grupos (Tabela 5).

TABELA 5. Agrupamentos da saúde psíquica

\begin{tabular}{|c|c|c|}
\hline Agrupamentos & $\mathbf{N}$ & Descrição \\
\hline Pessimistas & 16 & $\begin{array}{l}\text { Indivíduos apresentam sintomas graves de ordem física e psíquica, raros sentimentos } \\
\text { positivos e frequentes sentimentos negativos com relação ao trabalho. }\end{array}$ \\
\hline Ambivalentes & 22 & $\begin{array}{l}\text { Indivíduos que, embora apresentem sintomas graves de ordem física e psíquica, } \\
\text { manifestam também, em igual proporção, sentimentos positivos e negativos com relação } \\
\text { ao trabalho. }\end{array}$ \\
\hline Somatizadores & 20 & $\begin{array}{l}\text { Indivíduos que não apresentam sintomas psíquicos como estresse, desejo de morte ou } \\
\text { desconfiança do desempenho, mas sintomas graves psicossomáticos e do sono. Os } \\
\text { afetos positivos e negativos são baixos. }\end{array}$ \\
\hline Depressivos & 27 & $\begin{array}{l}\text { Indivíduos cujo único sintoma grave é o desejo de morte, apresentando-se sintomáticos } \\
\text { com relação aos outros fatores do QSG-60. Eles têm afetos positivos com relação ao } \\
\text { trabalho, com predominância daqueles que conduzem a um comportamento letárgico, e } \\
\text { apresentam sentimentos negativos da mesma ordem. }\end{array}$ \\
\hline Otimistas & 35 & $\begin{array}{l}\text { Indivíduos que não apresentam sintomas, possuem sentimentos positivos em relação ao } \\
\text { trabalho e raramente manifestam sentimentos negativos. }\end{array}$ \\
\hline
\end{tabular}

O agrupamento "Pessimistas" apresenta predomínio de sintomas depressivos e afetos negativos tanto ativadores como letárgicos acerca do trabalho e não apresentam sentimentos positivos. Isso significa que esses indivíduos tanto podem sofrer em silêncio como reagir com violência, não necessariamente física, às demandas do ambiente.

Os incluídos no agrupamento "Ambivalentes" têm afetos positivos para com o trabalho e ativação do comportamento, mas também apresentam afetos negativos. Em virtude disso, esses indivíduos podem demonstrar oscilações do comportamento. Provavelmente em função dos sentimentos positivos, observou-se redução da gravidade do sintoma depressivo representado pelo desejo de morte.

Nos "Somatizadores", destacam-se o distúrbio do sono, a redução dos afetos positivos e negativos e os sintomas psicossomáticos graves. A gravidade deste último indica ser provável haver dificuldade por parte desses indivíduos em lidar com os próprios sentimentos, cujo sintoma é uma defesa psíquica (Mello, 2002). O distúrbio do sono pode se relacionar a quadros depressivos, embora a ausência de desejo de morte torna mais provável que o distúrbio esteja ligado à atividade laboral ou aos sintomas psicossomáticos.

Nos "Depressivos", a depressão é representada pelo desejo de morte. Apesar de os afetos serem positivos, é provável que esses indivíduos aparentem ser pouco reativos ao meio, dificultando a percepção de que precisa de ajuda.

Por fim, nos "Otimistas", incluíram-se aqueles que frequentemente apresentam afetos positivos sobre o trabalho e não apresentam sintomas detectados pelos fatores do QSG-60.

A análise dos agrupamentos representativos da saúde psíquica dos profissionais de saúde nos hospitais evidenciou que os sujeitos que mantêm afetos positivos sobre o trabalho apresentam mais bem-estar e menos sintomas de agravamento da saúde comparados àqueles cujos afetos negativos predominam. Mostrou também que os afetos ativadores, que levam a possibilidades de reação dos sujeitos, mesmo quando negativos, contribuem para que se adaptem melhor ao meio.

\section{Saúde psíquica e condições de trabalho}

Para compreender a variância dos escores nos fatores das "Condições físicas e materiais", segundo a saúde psíquica dos profissionais da área da saúde participantes da pesquisa, aplicou-se ANOVA (Tabela 6). Constatou-se que as médias no fator "Aspectos psicobiológicos" diferem entre os grupos ( $\mathrm{F}=5,190$ para $\mathrm{p}=0,001$ ), mostrando que os participantes, segundo os agrupamentos de saúde psíquica, percebem os aspectos psicobiológicos de modo diferente. Portanto, os aspectos psicobiológicos, a exigência de esforço físico e os riscos de acidentes diferenciam os grupos de saúde psíquica. 
O teste pós-hoc Bonferroni mostrou que os aspectos psicobiológicos são mais percebidos pelos pessimistas $(\mathrm{M}=3,98 ; \mathrm{DP}=0,63)$, que apresentam mais sintomas graves de saúde psíquica e mais afetos negativos. $\mathrm{O}$ fator em análise é menos percebido pelos otimistas, que não apresentam sintomas psíquicos e têm sentimentos positivos em relação ao trabalho. A média no fator $(\mathrm{M}=3,04 ; \mathrm{DP}=0,84)$ se encontra em um intervalo que reflete que o profissional de saúde neste grupo se sente muitas vezes exposto ao fator, sendo que a média dos pessimistas se aproxima bastante da exposição frequente. Logo, essa percepção das condições de trabalho possui bastante impacto sobre a saúde psíquica dos participantes da pesquisa.

A "Exigência de esforço físico" foi outro fator das condições de trabalho que o teste Bonferroni apontou como percebido em níveis diferentes entre os agrupamentos. De acordo com esse teste, os pessimistas $(\mathrm{M}=3,25$; $\mathrm{DP}=0,70)$ percebem que o trabalho lhes exige mais esforço físico que os participantes dos agrupamentos 2,4 e 5. Essa percepção é menor entre os somatizadores $(M=2,34$; $D P=0,65)$, para quem o esforço físico parece ser menos percebido. Esse agrupamento é o que apresenta mais sintomas psicossomáticos, distúrbio do sono e inibição dos afetos com relação ao trabalho, tanto positivos como negativos.

TABELA 6. Percepção das condições físicas e materiais nos grupos de saúde psíquica

\begin{tabular}{|c|c|c|c|c|c|}
\hline & & $\mathrm{N}$ & Média & Desvio padrão & ANOVA \\
\hline \multirow{6}{*}{$\begin{array}{l}\text { (F1) Aspectos } \\
\text { psicobiológicos }\end{array}$} & Pessimistas & 16 & 3,98 & 0,63 & \multirow[t]{6}{*}{$F=5,190$ para $p<0,001$} \\
\hline & Ambivalentes & 22 & 3,61 & 0,83 & \\
\hline & Somatizadores & 20 & 3,45 & 0,71 & \\
\hline & Depressivos & 27 & 3,13 & 0,79 & \\
\hline & Otimistas & 35 & 3,04 & 0,84 & \\
\hline & Total & 120 & 3,36 & 0,84 & \\
\hline \multirow{6}{*}{$\begin{array}{l}\text { (F2) Espaço de } \\
\text { trabalho }\end{array}$} & Pessimistas & 16 & 2,39 & 0,76 & \multirow[t]{6}{*}{$\mathrm{F}=0,449$ para $\mathrm{p}=0,773$} \\
\hline & Ambivalentes & 22 & 2,14 & 0,87 & \\
\hline & Somatizadores & 20 & 2,19 & 0,64 & \\
\hline & Depressivos & 27 & 2,34 & 0,75 & \\
\hline & Otimistas & 35 & 2,19 & 0,70 & \\
\hline & Total & 120 & 2,24 & 0,74 & \\
\hline \multirow{6}{*}{$\begin{array}{l}\text { (F3) Aspectos } \\
\text { físico-químicos }\end{array}$} & Pessimistas & 16 & 2,07 & 0,62 & \multirow[t]{6}{*}{$\mathrm{F}=0,706$ para $\mathrm{p}=0,589$} \\
\hline & Ambivalentes & 22 & 1,95 & 0,69 & \\
\hline & Somatizadores & 20 & 1,82 & 0,55 & \\
\hline & Depressivos & 27 & 1,99 & 0,68 & \\
\hline & Otimistas & 35 & 1,82 & 0,56 & \\
\hline & Total & 120 & 1,92 & 0,60 & \\
\hline \multirow{6}{*}{$\begin{array}{l}\text { (F4) Exigência de } \\
\text { esforço físico }\end{array}$} & Pessimistas & 16 & 3,25 & 0,70 & \multirow[t]{6}{*}{$F=3,096$ para $p=0,018$} \\
\hline & Ambivalentes & 22 & 2,89 & 0,87 & \\
\hline & Somatizadores & 20 & 2,34 & 0,65 & \\
\hline & Depressivos & 27 & 2,91 & 0,80 & \\
\hline & Otimistas & 35 & 2,73 & 0,89 & \\
\hline & Total & 120 & 2,80 & 0,84 & \\
\hline \multirow{7}{*}{$\begin{array}{l}\text { (F5) Risco de } \\
\text { acidentes }\end{array}$} & Pessimistas & 16 & 2,39 & 0,86 & \multirow[t]{7}{*}{$F=2,740$ para $p=0,032$} \\
\hline & Ambivalentes & 22 & 1,85 & 0,82 & \\
\hline & Somatizadores & 20 & 1,83 & 0,51 & \\
\hline & Depressivos & 27 & 1,78 & 0,66 & \\
\hline & Otimistas & 35 & 1,75 & 0,59 & \\
\hline & Total & 120 & 1,88 & 0,70 & \\
\hline & Total & 120 & 1,88 & 0,70 & \\
\hline
\end{tabular}

Acerca do fator "Risco de acidentes", o teste Bonferroni mostrou que os participantes percebem o risco de forma desigual, sendo maior a percepção dos pessimistas $(M=2,39$; $D P=0,86)$ do que dos otimistas $(M=1,75$; $\mathrm{DP}=0,79)$. Os outros fatores são percebidos de forma semelhante pelos ambivalentes, pelos somatizadores e pelos depressivos. Esse é o fator que possui as menores médias, sendo que a mais alta $(M=2,39)$ corresponde à percepção entre raras vezes e algumas vezes na escala aplicada. Se não há percepção do risco, tende a haver menos cuidado com a prevenção.

$\mathrm{Na}$ dimensão das condições de trabalho - Processos e características do trabalho - a ANOVA demonstrou que há diferenças no entendimento dos grupos de saúde psíquica com relação ao fator "Complexidade e




$\mathrm{DP}=0,62)$ e "Estímulo à colaboração" $(\mathrm{M}=3,49 ; \mathrm{DP}=0,78)$, há homogeneidade na percepção dos participantes (Tabela 7).

TABELA 7. Percepção dos processos e características do trabalho nos grupos de saúde psíquica

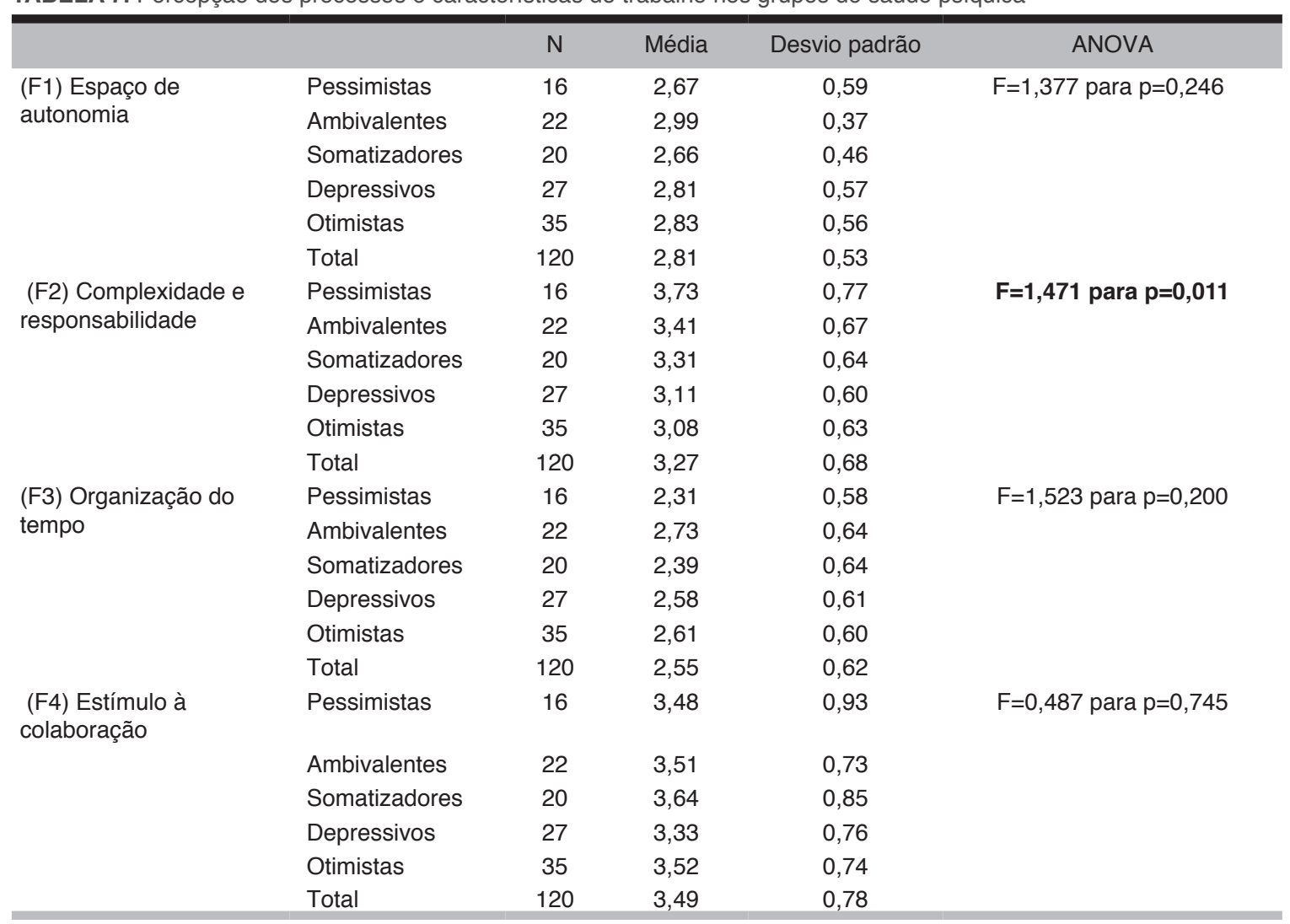

Para verificar como as percepções dos cinco agrupamentos da saúde psíquica eram hierarquizadas no fator "Complexidade e responsabilidade", foi aplicado o teste Bonferroni. Percebeu-se que os otimistas ( $M=2,83$; $\mathrm{DP}=0,56)$, composto por elementos assintomáticos, com afetos positivos com relação ao trabalho, sentem-se confortáveis com o nível de complexidade e responsabilidade que o trabalho lhes exige, sendo esta condição avaliada como adequada. Enquanto isso, os pessimistas $(\mathrm{M}=2,67 ; \mathrm{DP}=0,59)$, sintomáticos graves e com afetos negativos em relação ao trabalho, percebem as exigências do fator como desagradáveis ou inadequadas.

Em referência à variância da última dimensão das condições de trabalho - Ambiente sociogerencial segundo os agrupamentos de saúde psíquica dos participantes, a ANOVA indicou que há diferença na maneira como os profissionais de saúde percebem os fatores "Organização da atividade", "Violência" e "Ambiente conflitivo" (Tabela 8). O teste de Bonferroni, por sua vez, mostrou que os pessimistas $(\mathrm{M}=2,91 ; \mathrm{DP}=0,79)$ percebem que têm menos possibilidade de controlar suas atividades profissionais que os depressivos (M=1,94; $\mathrm{DP}=0,67)$, cujo único sintoma é o desejo de morte e que tem afetos positivos e negativos com relação ao trabalho. A percepção dos ambivalentes $(M=2,53 ; D P=1,20)$ também difere dos otimistas, sendo que aqueles participantes também percebem ter menos autonomia para organizar suas atividades profissionais.

Os pessimistas $(\mathrm{M}=2,05 ; \mathrm{DP}=1,23)$ percebem a exposição à violência diferentemente dos depressivos $(\mathrm{M}=1,18 ; \mathrm{DP}=0,32)$ e dos otimistas $(\mathrm{M}=1,52 ; \mathrm{DP}=0,68)$ (Tabela 8).

Em referência aos escores no fator "Ambiente conflitivo", o teste Bonferroni mostrou haver diferença entre a percepção dos pessimistas $(\mathrm{M}=2,77 ; \mathrm{DP}=1,06)$ e dos otimistas $(\mathrm{M}=1,87 ; \mathrm{DP}=0,68)$ acerca da existência de conflito no ambiente de trabalho. Para o primeiro agrupamento, o ambiente de trabalho é mais conflituoso do que para o segundo, que percebe que raramente o conflito se manifesta. Os demais fatores dessa dimensão são percebidos de modo homogêneo pelos participantes nos grupos (Tabela 8). 
TABELA 8. Percepção do ambiente sociogerencial nos grupos de saúde psíquica

\begin{tabular}{|c|c|c|c|c|c|}
\hline & & $\mathrm{N}$ & Média & $\begin{array}{l}\text { Desvio } \\
\text { padrão }\end{array}$ & ANOVA \\
\hline \multirow{6}{*}{$\begin{array}{l}\text { (F1) Organização da } \\
\text { atividade }\end{array}$} & Pessimistas & 16 & 2,91 & 0,79 & \multirow[t]{6}{*}{$\mathrm{F}=6,943$ para $\mathrm{p}<0,001$} \\
\hline & Ambivalentes & 22 & 2,53 & 1,20 & \\
\hline & Somatizadores & 20 & 2,62 & 0,59 & \\
\hline & Depressivos & 27 & 1,94 & 0,67 & \\
\hline & Otimistas & 35 & 1,91 & 0,68 & \\
\hline & Total & 120 & 2,28 & 0,88 & \\
\hline \multirow[t]{6}{*}{ (F2) Infraestrutura e pressão } & Pessimistas & 16 & 3,06 & 1,09 & \multirow[t]{6}{*}{$\mathrm{F}=0,677$ para $\mathrm{p}=0,609$} \\
\hline & Ambivalentes & 22 & 3,11 & 1,27 & \\
\hline & Somatizadores & 20 & 3,18 & 0,77 & \\
\hline & Depressivos & 27 & 3,08 & 1,16 & \\
\hline & Otimistas & 35 & 2,78 & 0,85 & \\
\hline & Total & 120 & 3,01 & 1,03 & \\
\hline \multirow{6}{*}{$\begin{array}{l}\text { (F3) Oferta de informações } \\
\text { de saúde }\end{array}$} & Pessimistas & 16 & 2,34 & 1,33 & \multirow[t]{6}{*}{$F=1,618$ para $p=0,174$} \\
\hline & Ambivalentes & 22 & 2,78 & 1,23 & \\
\hline & Somatizadores & 20 & 1,87 & 0,90 & \\
\hline & Depressivos & 27 & 2,52 & 1,24 & \\
\hline & Otimistas & 35 & 2,46 & 1,23 & \\
\hline & Total & 120 & 2,42 & 1,21 & \\
\hline \multirow[t]{6}{*}{ (F4) Discriminação } & Pessimistas & 16 & 1,11 & 0,28 & \multirow[t]{6}{*}{$F=1,489$ para $p=0,210$} \\
\hline & Ambivalentes & 22 & 1,32 & 0,61 & \\
\hline & Somatizadores & 20 & 1,10 & 0,28 & \\
\hline & Depressivos & 27 & 1,08 & 0,25 & \\
\hline & Otimistas & 35 & 1,19 & 0,37 & \\
\hline & Total & 120 & 1,16 & 0,38 & \\
\hline \multirow[t]{6}{*}{ (F5) Participação } & Pessimistas & 16 & 2,50 & 0,48 & \multirow[t]{6}{*}{$F=1,453$ para $p=0,221$} \\
\hline & Ambivalentes & 22 & 2,76 & 0,96 & \\
\hline & Somatizadores & 20 & 2,43 & 0,73 & \\
\hline & Depressivos & 27 & 2,46 & 0,80 & \\
\hline & Otimistas & 35 & 2,26 & 0,71 & \\
\hline & Total & 120 & 2,46 & 0,78 & \\
\hline \multirow[t]{6}{*}{ (F6) Violência } & Pessimistas & 16 & 2,05 & 1,23 & \multirow[t]{6}{*}{$F=5,209$ para $p<0,001$} \\
\hline & Ambivalentes & 22 & 1,59 & 0,74 & \\
\hline & Somatizadores & 20 & 1,65 & 0,42 & \\
\hline & Depressivos & 27 & 1,18 & 0,32 & \\
\hline & Otimistas & 35 & 1,39 & 0,46 & \\
\hline & Total & 120 & 1,52 & 0,68 & \\
\hline \multirow[t]{6}{*}{ (F7) Ambiente conflitivo } & Pessimistas & 16 & 2,77 & 1,06 & \multirow[t]{6}{*}{$F=4,160$ para $p=0,003$} \\
\hline & Ambivalentes & 22 & 2,39 & 1,30 & \\
\hline & Somatizadores & 20 & 2,63 & 1,00 & \\
\hline & Depressivos & 27 & 1,91 & 0,88 & \\
\hline & Otimistas & 35 & 1,87 & 0,68 & \\
\hline & Total & 120 & 2,22 & 1,02 & \\
\hline
\end{tabular}

\section{CONSIDERAÇÕES FINAIS}

Nesta pesquisa, foram relacionados os fatores das dimensões das condições de trabalho com os escores de saúde psíquica dos participantes. As análises mostraram variâncias conjuntas que corroboram a noção de que a saúde psíquica sofre influência das condições laborais, sobretudo no que diz respeito aos aspectos psicobiológicos, à exigência de esforço físico e ao risco de acidentes, na dimensão "Condições físicas e materiais", da complexidade e responsabilidade, avaliada na dimensão "Processos e características do trabalho" e dos fatores "Organização da atividade", "Violência" e "Ambiente conflitivo", da dimensão "Ambiente sociogerencial".

Dessa maneira, há uma tendência de que, quanto mais desfavoráveis as condições de trabalho, mais deteriorada será a saúde psíquica e os afetos com relação ao trabalho, repercutindo novamente no ambiente de trabalho. Portanto, ações de melhoria dessas condições precisam ser estabelecidas para resultar no efeito inverso, proporcionando o aumento dos afetos positivos e sua expressão e a redução dos sintomas psíquicos. 
Se considerarmos que as significações do trabalho na vida de um trabalhador são construídas coletivamente e que as relações interpessoais estabelecidas são determinantes nesse processo, podemos compreender, a partir dos resultados encontrados, que há risco no trabalho dos profissionais de saúde estudados. Esse risco é causado por múltiplos fatores que configuram-se na relação dialética do trabalhador com seu contexto de trabalho, do qual fazem parte as condições materiais e físicas, a organização dos processos e as relações entre os atores sociais.

Portanto, a contribuição epistemológica deste estudo está em evidenciar que os pressupostos da abordagem psicossociológica são adequados para a avaliação da saúde psíquica em relação às condições de trabalho de profissionais de saúde, por considerarem a relação entre o contexto de trabalho e a saúde psíquica.

Registra-se que este estudo encontrou limitações, como a possibilidade de generalização do estudo para outras categorias, uma vez que, partindo do contexto, seu entendimento é indissociável da compreensão de como se configura a saúde psíquica em um grupo de trabalhadores. Diante dessas limitações, sugere-se ampliar este estudo avaliando as condições de trabalho com relação à saúde psíquica em outras categorias profissionais e contextos diversos, e fazer estudos comparativos entre profissionais de setores públicos e privados.

Por fim, registra-se também que o tamanho da amostra foi um aspecto limitador, impossibilitando, por exemplo, examinar as evidências de validade e consistência dos questionários estruturados utilizados exclusivamente para tal amostra. Entretanto, as características de funcionamento dos hospitais não permitiram a obtenção de amostra mais extensa em tempo hábil.

\section{REFERÊNCIAS}

Albrecht, K. (1990). O gerente e o estresse: faça o estresse trabalhar para você. (2a ed.) Rio de Janeiro: Zahar.

Almeida N. Filho, \& Jucá, V. (2002). Saúde como ausência de doença: crítica à teoria funcionalista de Christopher Boorse. Ciência \& Saúde Coletiva, 7(4), 879-889.

Álvaro, J. L. (1995). Psicologia social: perspectivas teóricas y metodológicas. Madrid: Siglo XXI de España Editores.

Álvaro, J. L., Garrido, A., \& Torregrossa, J. R. (2007). Psicologia social aplicada. Madrid: Mc-Graw Hill/Interamericana de España.

Batistella, C. (2007). Abordagens contemporâneas do conceito de saúde. In A. F. Fonseca, A. M. D. Corbo (Org.). O território e o processo saúde-doença (pp. 51-86). Rio de Janeiro: EPSJV/Fiocruz.

Benavides, F. G., García, A. M., \& Ruiz-Frutos, C. (2000). La salud y sus determinantes. In F. G. Benavides, C. Ruiz-Frutos, \& A. M. García (Orgs.). Salud laboral. Conceptos y técnicas para la prevención de riesgos laborales (pp. 37-48). Barcelona: Masson.

Benevides-Pereira, A. M. (2002). Bournout: quando o trabalho ameaça o bem-estar do trabalhador. São Paulo: Casa do Psicólogo.

Blanch, J. M. (2003). Condiciones de Trabajo. In J. M. Blanch, M. J. Espuny, C. Gala, \& A. Martín (Orgs.). Teoría de las relaciones laborales. Fundamentos (pp. 42-44). Barcelona: Editorial UOC.

Borges, L. O., Alves-Filho, A., Costa, M. T. P., \& Falcão, J. T. R. (no prelo). Condições de trabalho. In P. F. Bendassolli \& J. E. Borges-Andrade (Org.), Dicionário Brasileiro de Psicologia do Trabalho e das Organizações. São Paulo: Casa do Psicólogo.

Borges, L. O., Costa, M. T., Alves-Filho, A., Souza, A. L. R., Leite, C. P. R. L. A., Falcão, J. T. R. (2013). Questionário de condições de trabalho: reelaboração e estruturas fatoriais em dois grupos ocupacionais. Avaliação Psicológica, 12(2), 213225. Recuperado de http://www.redalyc.org/articulo.oa?id=335027505012

Canguilhem, G. (2006). O normal e o patológico. Rio de Janeiro: Forense Universitária (Original publicado em 1966).

Castelhano, L. M. (2005). O medo do desemprego e a(s) nova(s) organizações de trabalho. Psicologia \& Sociedade, 17(1), $17-28$. doi:10.1590/S0102-71822005000100003

Coelho, M. T. A. D., \& Almeida Filho, N. (2002). Conceitos de saúde em discursos contemporâneos de referência científica. História, Ciências, Saúde, 9(2), 315-333. doi:10.1590/S0104-59702002000200005

Conselho Federal de Medicina. (1998). Resolução CFM 1488/1998. Recuperada de http://www.portalmedico.org.br/resolucoes/ cfm/1998/1488_1998.htm.

Constituição da República Federativa do Brasil de 1988. (1988). Brasília.

Costa, J. R. A., Lima, J. V., \& Almeida, P. C. (2003). Stress no trabalho do enfermeiro. Revista Escola de Enfermagem, 37(3), 6371. 
Elias, M. A., \& Navarro, V. L. (2006). A relação entre o trabalho, a saúde e as condições de vida: negatividade e positividade no trabalho das profissionais de enfermagem de um hospital escola. Revista Latino-Americana de Enfermagem, 14(4), 514-25. Recuperado em 14 novembro, 2009, de www.eerp.usp.br/rlae. doi: 10.1590/S0104-11692006000400008

Engels, F. (2008). A situação da classe trabalhadora na Inglaterra. São Paulo: Boitempo Editorial (Original publicado em 1845).

European Working Conditions Observatory. (2006). Annual review of working conditions in the EU: 2005-2006. Recuperado em 04 de março de 2007 de http://www.eurofound.europa.eu/pubdocs/2006/20/en/1/ef0620en.pdf

Fontes, V. (2001). História e Verdade. In M. Chiavatta, \& G. Frigotto (Orgs.). Teoria educação no labirinto do capital (pp. 130-155). Petrópolis: Vozes.

Garrido, A. (Org). (2005). Sociopsicologia del trabajo. Barcelona: Editorial UOC.

Gouveia V. V., Fonseca P., Lins, S. B., \& Gouveia, R. (2008). Escala de Bem-estar Afetivo no Trabalho (JAWS): evidências de validade fatorial e consistência interna. Psicologia: Reflexão e Crítica, 21, 464-73. doi:10.1590/S0102-79722008000300015

Gouveia, R. S. V., Lins, Z. M. B., Lima, T. J. S., Freires, L. A., \& Gomes, A. I. A. S. B. (2009). Bem-Estar afetivo entre profissionais de saúde. Revista Bioética, 17(2), 267-280. Recuperado em 19 março, 2010, de http://revistabioetica.cfm.org.br/index.php/ revista_bioetica/article/viewFile/166/171

Joyce, K., Pabayo, R., Critchley, J. A., \& Bambra, C. (2010). Flexible working conditions and their effects on employee health and wellbeing. Cochrane Database of Systematic Reviews, 2. Recuperado em 12 março, 2010 de http://www3.interscience.wiley. com/homepages/106568753/CD008009.pdf. doi: 10.1002/14651858.CD008009.pub2

Laurell, A. C., \& Noriega, M. (1989). Processo de produção e saúde. Trabalho e desgaste operário. São Paulo: Cebes-Hucitec.

Leriche, R. (1940). La Chirurgie de la douleur. Paris. Masson.

Marx, K., \& Engels (1981). A ideologia alemã. Lisboa: Editorial “Avante”!(Original publicado em 1846)

Mauro, M. Y. C., Paz, A. F., Mauro, C. C. C., Pinheiro, M. A. S., \& Silva, V. G. (2010). Condições de trabalho da enfermagem nas enfermarias de um hospital universitário. Escola Anna Nery, 14(2), 244-252. Recuperado em 20 setembro, 2012, de http://www.scielo.br/scielo.php?script=sci_arttext\&pid=S1414-81452010000200006\&lng=en\&tIng=pt. doi:10.1590/S141481452010000200006

McCue, J. D. (1982). The effects of stress on physicians and theirmedical practice. New England Journal of Medicine, 306, 45863.

Mello, F., Jr. (2002). Concepção Psicossomática: Visão Atual. São Paulo: Casa do Psicólogo.

Merhy, E. E. (1999). O ato de governar as tensões constitutivas do agir em saúde como desafio permanente de algumas estratégias. Ciências \& Saúde Coletiva, 4, 305-29. doi:10.1590/\$1413-81231999000200006

Ministério da Saúde. (2001). Doenças relacionadas ao trabalho: manual de procedimentos para os serviços de saúde. Recuperado em 12 janeiro, 2008, de http://www.portal.saúde.gov.br.

Ministério do Trabalho e Emprego (2005). NR 32 - Segurança e saúde no trabalho em serviços de saúde. Recuperado de http:// portal.mte.gov.br/data/files/8A7C816A350AC8820135161931EE29A3/NR-32\%20(atualizada\%202011).pdf em 11 de janeiro de 2012.

Mozer, A. D., \& Kherig, R. (2006). O conceito de saúde e seus desdobramentos nas várias formas de atenção à saúde do trabalhador. Fisioterapia em Movimento, 19(4), 89-97.

Murofuse N. T., Abranches S. S., \& Napoleão A. A. (2005). Reflexões sobre estresse e Burnout e a relação com a enfermagem. Revista Latino-Americana de Enfermagem, 13(2),255-261. doi:10.1590/S0104-11692005000200019

Organização Mundial da Saúde. (1948). Preamble to the constitution of the world health organization. In Official records of the world health organization, no 2. Recuperado de http://policy.who.in/cgi-bin/om_isapi.dll/infobase=Basicdoc\&softpage=Browse_Frame_Pg42.Parsons, T. (1951). The social system. Glencoe: Free Press.

Organização Mundial da Saúde. (2001). Relatório sobre a saúde no mundo 2001: Saúde mental: nova concepção, nova esperança. Recuperado em 10 março, 2010, de http://www.abebe.org.br/wp-content/uploads/oms2001.pdf.

Parsons, T. (1951). The social system. Glencoe, IL: Free Press.

Pasquali, L., Gouveia, V. V., Andriola, W. B. Miranda, F. J., \& Ramos, A. L. M. (1994). Questionário de Saúde Geral de Goldberg (QSG): Adaptação brasileira. Psicologia: Teoria e Pesquisa, 10(3), 421-438.

Peiró, J. M., \& Prieto, F. (2002). Tratado de psicología del trabajo. La actividad laboral em su contexto. Madri: Ed. Síntesis.

Pietro, C. (1994). Trabajadores y condiciones de trabajo. Madri: Ediciones HOAC. 
Pires, D. (2000). Reestruturação produtiva e consequências para o trabalho em saúde. Revista Brasileira de Enfermagem, 53, 251-63. doi:10.1590/S0034-71672000000200010

Pitta, A. (1990). Hospital dor e morte como ofício. São Paulo. Hucitec.

Politzer, G., Besse, G., \& Caveing, M. (1970). Princípios Fundamentais da Filosofia. São Paulo: Hemus Livraria.

Ramos, J., Peiró, J. M., \& Ripoll, P. (2002). Condiciones de trabajo y clima organizacional. In J. M. Peiró, \& F. Prieto (Org.), Tratado de psicología del trabajo: la actividad laboral en su contexto (pp. 37-92). Madrid: Síntesis Psicología.

Sanz Yagüez, F., \& López Corbalán, J. C. (1999). Abuso de sustancias psicoactivas entre los profesionales de la salud. Revista española de anestesiología y reanimación, 46(8), 354-8.

Sartre, J. P., \& Ferreira, V. (1961). O existencialismo é um humanismo. Lisboa: Editorial Presença.

Schraiber, B. L., Peduzzi, M., Sala, A., Nemes, M. I. B., Castanhera, E. L. R., \& Kon, R. (1999). Planejamento, gestão e avaliação em saúde: identificando problemas. Ciências e Saúde Coletiva, 4, 221-42. doi: 10.1590/S1413-81231999000200002

Silva, C. O. (2002). Vida de hospital: a produção de uma metodologia para o desenvolvimento da saúde do profissional de saúde. Tese de doutorado, Fundação Oswaldo Cruz, Rio de Janeiro, RJ, Brasil.

Warr, P. (1987). Work, unemployment, mental health. New York: Oxford University Press.

Zanelli, J. C., Bastos, A. V. B., \& Rodrigues, A. C. A. (2014). Campo profissional do psicólogo em organizações e no trabalho. In J. C. Zanelli, J. E. Borges-Andrade, \& A. V. B. Bastos (Orgs.). Psicologia, Organizações e Trabalho no Brasil (2ª ed., pp. 549582). Porto Alegre: Artmed. 